\title{
A METHOD FOR THE DETERMINATION OF CARBONATES IN SOILS.
}

\author{
By ARTHUR AMOS, B.A., \\ Rothamsted Experiment Station.
}

THE methods usually employed for estimating calcium carbonate in soil are all subject to more or less serious practical difficulties when the percentage of lime falls below 0.5 .

In gravimetric methods, in which the carbon dioxide set free by acid has first to be boiled out of solution and then perfectly dried before absorption by potash, a very small weight of carbon dioxide is estimated by the gain in weight of a comparatively heavy absorption apparatus.

The volumetric method of Scheibler also cannot be used for soil containing only 0.5 per cent. of lime because all the carbon dioxide remains in solution in the decomposing acid.

The method devised by Hall and Russell ${ }^{1}$ (in which the lime is decomposed in an exhausted apparatus, and the carbon dioxide set free calculated from three readings of pressure, taken (1) before the addition of acid, (2) on equilibrium being established after the acid is added, (3) on equilibrium being established when the gas is allowed to expand into a further exhausted and known volume) gives very accurate results; but much shaking is required in order to overcome the supersaturation of the decomposing acid with the carbon dioxide, and hence each determination occupies a considerable time, and leakages are apt to occur unless the taps are very perfect.

Brown and Escombe ${ }^{2}$ in the course of their work on carbon assimilation of plants have shown that, by a modification of Hart's method of double titration for estimating sodium carbonate in the presence of

1 Trans. Chem. Soc. 1902, Vol. Lxxxi. p. 81.

2 Phil. Trans. Roy. Soc. 1900, Vol. cxcur. pp. 289-291. 
sodium hydrate, quantities of carbon dioxide, varying from 1 to 100 c.c. and contained in 100 c.c. of 4 per cent. sodium hydrate solution, can be determined within 2 c.c. of the quantity added.

Whilst making use of this method for determining the varying amounts of carbon dioxide in the atmosphere it was suggested to me by Mr A. D. Hall, M.A., that a modification of this might be employed for determining calcium carbonate in soil, which led to the following method being devised.

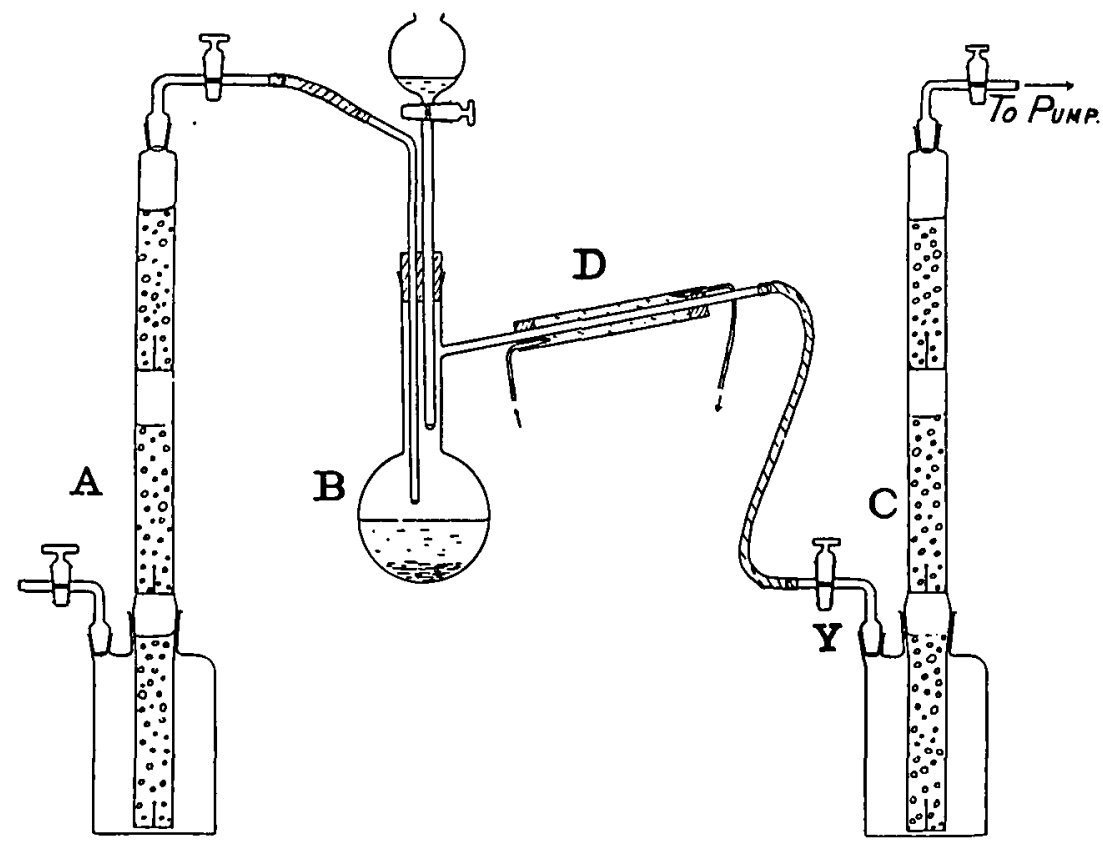

The apparatus as shown in the diagram consists of :-

A. A Reiset absorption apparatus containing 100 c.c. of a 4 per cent. sodium hydrate solution for washing the air free from carbon dioxide.

B. A Jena flask, in which the soil to be treated is put; the flask is provided with a side tube.

C. A second Reiset apparatus containing 100 c.c. of a per cent. sodium hydrate solution for absorbing the carbon dioxide set free in $\mathrm{B}$.

D. A condenser for cooling the air passing into $C$ to prevent the lubricant of the inlet tap being melted.

Procedure:-A weighed quantity of soil is put into the flask $B$ and shaken up with 75 c.c. of carbon dioxide free water (any weight of soil 


\section{Method for Determination of Carbonates in Soil}

may be used up to 50 grams, containing not more than 0.5 gram of lime); the apparatus is now connected up as in the figure except that the end $Y$ of the rubber tube is connected directly with the pump, and a stream of air is drawn through the apparatus, so that all the atmospheric carbon dioxide is swept out ; the pump is stopped and the Reiset $\mathrm{C}$ introduced between $\mathrm{Y}$ and the pump, and the pump started again, so that a steady stream of air free from carbon dioxide is drawn through the apparatus. 20 c.c. of strong hydrochloric acid are now run into B by means of the dropping funnel, and the contents of B gradually brought to the boiling point; the boiling is continued for 20 minutes to ensure all the carbon dioxide being swept into the Reiset $\mathrm{C}$.

The titration is carried out in the lower part of the Reiset apparatus, into which the contents of the absorbing tube are washed, phenol-phthalein is added and normal hydrochloric acid run in until the pink colour begins to fade, then decinormal hydrochloric is run in until the colour is completely discharged, the reading of the decinormal hydrochloric acid is now taken, methyl-orange is added, and the titration continued until the methyl-orange shows an acid reaction; a second reading of the hydrochloric acid is now taken.

The difference between the two readings gives the volume of decinormal hydrocbloric acid required in the equation,

$$
\mathrm{NaHCO}+\mathrm{HCl}=\mathrm{NaCl}+\mathrm{H}_{2} \mathrm{O}+\mathrm{CO}_{2},
$$

and hence we get the weight of calcium carbonate originally present in the soil.

The object of employing normal hydrochloric acid in the first part of the titration is to prevent unnecessary dilution, but the liquid must be kept in motion so that the acid is never in excess at any point with consequent evolution of $\mathrm{CO}_{2}$.

The presence of aluminium and iron has been shown to interfere with the sharpness of the second colour reaction, consequently the sodium hydrate employed must be prepared from metallic sodium.

In order to get an exact reading at the end of the second titration a standard solution of methyl-orange, contained in a vessel of the same shape and size, and having an arbitrary tint of acidity should be employed, with which to compare the tint.

These precautions are some of those suggested by Brown and Escombe.

A preliminary blank experiment must be carried out in order to correct for:- 
(i) The amount of carbon dioxide originally present in the 100 c.c. of sodium hydrate used in the Reiset.

(ii) The $\mathrm{CO}_{2}$ contained in the air in the Reiset apparatus $\mathrm{C}$ before the soda solution is run into it.

(iii) Any $\mathrm{CO}_{3}$ in solution in the 20 c.c. of acid employed for decomposing the lime.

The following table gives the results of a series of experiments carried out for the purpose of testing the accuracy of the method; weighed quantities of ground Iceland spar, or a known volume of a solution of sodium carbonate, were treated by the above method, and the weight of calcium carbonate or sodium carbonate calculated from the carbonic acid absorbed.

\begin{tabular}{|c|c|c|}
\hline $\begin{array}{c}\text { No. of } \\
\text { Experiment }\end{array}$ & \begin{tabular}{c} 
Weight of $\begin{array}{c}\mathrm{CaCO}_{3} \text { taken } \\
\text { (grams) }\end{array}$ \\
\hline 1
\end{tabular}$\quad \begin{array}{c}\text { Weight of } \mathrm{CaCO}_{3} \text { found } \\
\text { (grams) }\end{array}$ \\
\hline 2 & 0.057 & 0.056 \\
3 & 0.105 & 0.104 \\
4 & 0.242 & 0.245 \\
5 & 0.542 & 0.541 \\
6 & 0.564 & 0.559 \\
& 25 c.c. $=0.0250 \mathrm{Na}_{2} \mathrm{CO}_{3}$ & $0.0248 \mathrm{Na}_{2} \mathrm{CO}_{3}$ \\
\hline
\end{tabular}

The absorption of carbon dioxide in the Reiset apparatus is very perfect; the rate at which air nay be passed through and still be completely freed from carbon dioxide is only limited by the overflow of the sodium hydrate solution.

When a series of calcium carbonate estimations have to be made the method is reliable and quick; one determination lasting about 45 minutes; but when only isolated determinations have to be made, the method of Hall and Russell is more expeditious because of the time involved in the preparation of solutions and other preliminaries.

The above method was employed for the estimation of calcium carbonate in some soils taken from the Royal Agricultural Society's farm at Woburn; the following table shows the results obtained: the first column gives the name of the plot from which the soil was taken; the second gives the weight of soil employed; the third gives the volume of acid in duplicate experiments used in the titration; the fourth gives the volume of acid required in a blank experiment; the fifth gives the calculated percentages of calcium carbonate. 


\section{Method for Determination of Carbonates in Soils}

It will be seen that the duplicates agree except in one instance (in the first depth of plot 3 of the Rotation field), in which one low figure was obtained.

Plot $2 \mathrm{~A}$ in the Stackyard field is the well-known 'acid' plot, which has been continuously manured with ammonium salts, the fresh soil of this plot gives an acid reaction with litmus.

The percentage of calcium carbonate found in the first depth of this soil $(0.053)$ was lower than that found in the first depths of any of the other plots but yet seemed inconsistent with its acid reaction.

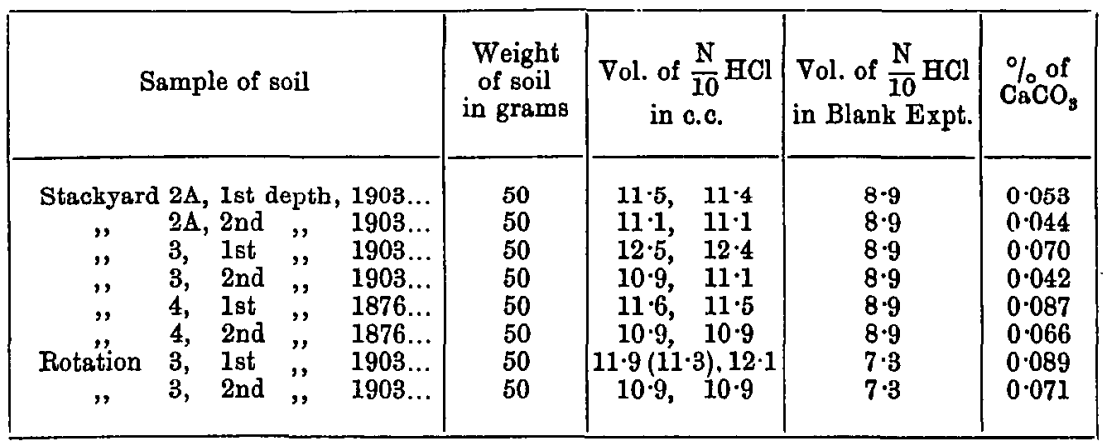

It was thought that this might be due to carbon dioxide being occluded by the soil particles; in order to test this, 50 grams of soil were digested with hydrochloric acid, filtered, washed and then stirred up with some water saturated with carbon dioxide, and allowed to dry in the air at the ordinary temperature; the carbon dioxide was then estimated by the method, when it was found that no carbon dioxide had been occluded.

A further experiment was carried out to test this point: 50 grams of plot $2 \mathrm{~A}$ were placed in the flask of the apparatus with water only, no hydrochloric acid being added, the mixture was boiled, and any gas evolved passed through the Reiset tower; it was found that no carbon dioxide had been absorbed; hence we must conclude that the calcium carbonate is genuinely present, and its presence will explain the fact that when this soil is stirred up with water, and the mixture kept for some weeks, the acidity slowly disappears, owing to the interaction between the acid and the carbonate. 